\title{
Smart Home based Health Care Systems for Elderly People
}

\section{Janani* and M Meenalochini}

Assistant Professor, Department of CSE, Jai Shriram Engineering College, Tirupur, TN, India

*Corresponding Author: M Janani, Assistant Professor, Department of CSE, Jai Shriram Engineering College, Tirupur, TN, India.

Received: June 13, 2019; Published: July 19, 2019

DOI: $10.31080 /$ ASNH.2019.03.0374

\begin{abstract}
Rapid evolution of sensing technology and increasing power computation results in the emergence of smart environments with smart health services. Smart environments per day can generate hundreds of thousands transactions and storage over a long term seems to be arduous. Thereby, smart environment big data is utilized which stores large volume of data sets, both structured and unstructured. Health care services are recent and challenging aspects in analytics and sensor technology. People migration from rural area to urban area affects the health care services in vast ratio. Due to migration and developing technology, cities in the world are investing on digital transformation which aims to provide people a healthier environment. Health care application is merely based on activity recognition of people and includes wearable or ambient sensors to gather information related to health and human activity. Consumption of energy is analyzed along with activity pattern of human by determining the level of appliances usage which relates human activity. The pattern determined is used to track elderly people living all alone. Activity monitored can be learned only from appliances and its usage of time. The proposed model detects appliance usages in particular with reliable accuracy. Mining is done purely based on absolute prediction along with clustering models.
\end{abstract}

Keywords: Smart Environment; Healthcare Service; Human Activity Pattern; Prediction

\section{Introduction}

Data mining is the computing process of discovering patterns in large data sets involving machine learning, statistics and database systems. It is a process of extracting data patterns by applying intelligent methods and transforms it into an understandable structure for further use. Data mining parameters includes sequence or path analysis, classification, clustering and forecasting. The concept of data mining includes statistics, artificial intelligence and machine learning.

The smart environment includes smart home, smart city, smart meter, smart parking, smart governance and sensors. Smart environments provide a way to respond to the needs of the residents in a context aware manner. A smart environment is equipped with different types of sensors which allow the system to collect data on inhabitant activities and environmental situations. Activity at smart home can be collected using ambient sensors such as infrared motion sensors to track the motion of residents around home. Additionally, ambient sensors is used for sensing in the form of temperature sensors, pressure sensors, contact switch sensors and smart power meter which provides context information.

Smart city is an urbanized area where multiple sectors cooperate to achieve sustainable outcomes. This is done through the analysis of contextual and real- time information. Moreover, a smart city can provide intelligent responses to different kinds of daily needs, including livelihood of people, security systems, public transportation and environment, public health and industrial and commercial activities. Smart city data faces multiple challenges due to its volume, velocity and variety. The data is unstructured because it may be in the form of audio, images, log files, tweets, text etc and it has to be integrated with structured data.

The sources of data could be legacy sources, new technology sources, IoT devices or manual entry done by human beings. The IoT sensors may not be working at some places, giving faulty or missing data. Thus, lot of preprocessing needs to be done on such data. Smart city data becomes Big Data when it comes to technol- 
ogy. Big data is a term that describes large volume of data, both structured and unstructured.

Big data is collection of data sets of large or complex data that the traditional data processing is inadequate to deal with. Challenges of big data are capturing data, data storage, data analysis, search, sharing, transfer, visualization, querying updating and information privacy. Major challenges include information sharing, privacy, security, new data formats and quality of data. Therefore, it poses all those challenges which big data faces in addition, it faces challenges related to security and privacy as well. Smart meters are installed to monitor the activities at home. Loads of appliance is monitored whic is used to know the individual appliance usage. Smart meter along with smart power plugs are used to collect data which tracks people activity regularly by classifying them in the appliances usage consumption.

Big data applications in healthcare organizations provides significant benefits which include detecting diseases at an early stage and prescribed more easily and effectively. Advancement in tool and technology of smart cities improves healthcare services. The infrastructure and technology of smart cities reconstructs often by thinking beyond existing healthcare systems. Telemedicine also recreates a new and comfortable ubiquitous concept called smart health. Moreover, smart health integrates ideas from ubiquitous computing and ambient intelligence applied to predictive, personalized, preventive and participatory healthcare systems. Smart health is strongly connected to the concepts of wellness and wellbeing which includes large volume of data collected from biomedical sensors. Thereby big data has genomic driven, payer provider and social media data actuators to observe and predict patient's physical and mental conditions.

Smart health is a nascent but promising field of study at the intersection of medical informatics, public health and also business, alluding to intelligent healthcare services or enhanced cognitive capabilities through the IoT. Healthcare service is affected due to migaration of people to city sides where digital transformation is an recent urge. To provide healthier environment, homes are are entirely organized by smart devices which makes the city smart. Smart city would generates millions and millions of data throughout which should be refined and should make as useful structured data.

\section{Literature Review}

MuhammadAftab and Chi-KinChau (2017) proposed Smart power plugs, a notable cyber physical systems for tracking and controlling appliance behavior. In traditional smart plugs, advanced automated features such as online learning, classification and diagnosis of appliance behavior is not included. The existing algorithm for classification and clustering are not designed with storage and computation limitations. Hence, Stand alone smart plugs are developed for providing efficient classification and tracking. To track the performance and sensing of evenly distributed tasks by the local memory, Internet of Things (IoT) framework is used [1].

Jianguo Hao, Abdenour Bouzouane and Sebastien Gaboury (2017) proposed a paper in which prediction of activity is done using hardware and software with support of smart homes. Smart homes are equipped with intelligent services for providing best living environment of user. Human behaviour are monitored using ambient sensor and multi model interactions happened in smart homes are mined [2]. Feedbacks are generated then there for elderly people by recognized behaviour.

A Smart City is an urban innovation aimed to improve the quality of life. Smart city employs technology to bring about development on social and economic front. The various applications of Smart meters are energy management, Green buildings, Solar usage, Smart parking, Intelligent traffic management, Waste management, Crime monitoring and management, Water quality monitoring and leakage identification. Creating walk able localities and making areas less vulnerable to disasters for smart Governance. Data captured in the smart city initiative is used to improve the livability of the city. This data includes the government data as well as social data. Social data is used to analyze the sentiments of the citizens during important city events [3].

Anish Jindal, Amit Dua and Neeraj Kumar (2017) proposed a paper on health care. Advancements in information and communication technology increases number of user availing remote healthcare applications. The data collected about the patients in these applications varies with respect to volume, velocity, variety, veracity, and value. To process such a large collection of heterogeneous data is one of the biggest challenges that need a specialized approach. To address this issue, a new fuzzy rule-based classifier is used for handling big data along with cloud based infrastructure 
named Healthcare-as-a-Service [4]. The Proposed work is done by Abdulsalam Yassine, Shailendra Singh and Atif Alamri (2017) deals with Healthcare service, which is one of the most challenging aspects that are greatly affected by the vast influx of people to city centers. People in the cities are moving towards healthier environment which will be a part of digital transformation in smart city services [5]. Mass volume of data generated from the smart home will give further enhnace into smart cities.

The work has been proposed by Jaganathan Venkatesh, Baris Aksanli and Christine S. Chan (2017) where smart health is studied based on IoT. The Internet of Things (IoT) envisions to create the smart connected cities composes of ubiquitous environmental and user sensing with distributed, low capacity computing. Provides a ample information regarding the citizens in various smart environments. People-centric information can be leveraged by providing the smart city infrastructure to improve smart health applications [6].

The work has been proposed by Md Ileas Pramanik, Raymond Y.K. Lau , Haluk Demirkan and Md Abul Kalam (2017). Recent technology development in Big data leads to the growth of Information and communication technology a vast development. Technology development brings in the Mobile computing where communication plays the major role and even ubiquitous computing provides opportunity for many government and private sectors especially in healthcare industries. Big data seems to be a back bone of healthcare industry. Three-dimensional structure of a paradigm shift is used and three broad technical branches (3T) are extracted contributing to the promotion of healthcare systems.

The work is carried out by Min Chen, Yixue Hao, Kai Hwang, Lu Wang and Lin Wang (2017). Big data growth in biomedical and healthcare area provides analysis of healthcare data which helps in disease detection of elder people.. When the prediction data goes wrong the healthcare details becomes incomplete seams to be useless. Since each diseases have unique symptoms and measures to be carried out. Even healthcare data can be an outlier when there is no active prediction. Machine learning algorithms is used for effective prediction and mining [8].

Emerging Cyber-Physical Systems (CPS) is proposed by M. Shamim Hossain and Md. Abdur Rahman (2017), for providing services for elderly people using smart home. CPS users interactions are collected and sensed from smart home. Interaction help in monitoring energy efficiency. Proposed work is energy-efficient system for monitoring elderly people with uses of technologies like cloud computing and big data [9]. Smart grid based data collection is proposed by Yuan Hong, Wen Ming Liu and Lingyu Wang (2017). Power usage data is collected through smart meters. Smart meter dtat for appliances are differentiated in the means of cost and load. Smart meter reading may vary inbetween the on and off status [10].

Anthony Faustine, Nerey Henry Mvungi, Shubi Kaijage and Kisangiri Michael (2017) carried out the urbanization towards developing countries considering big bulidings and high power consumption areas to measure the energy the energy efficiency. Continous monitoring of appliances power consumption in realtime is not easy task. There needs a aggregate power montoring system in smart home[11].

Classification done for smart cities is described by Victoria Moreno and Fernando Terroso (2016). Smart cities are the application of big data which acts as a major research technology. These contributions are the design and instantiation of an IoT-based architecture for applications of smart cities, management of energy in smart buildings and the extension of the data analysis for detection of urban patterns which is used to improve public transport applied to the public tram service [12].

Mining and usage patterns are proposed by Daniel Schweizer and Michael Zehnder (2016). Usage patterns based preferences are learned to allow smart home in achieving energy savings. Frequent sequential pattern mining algorithm is used for real-time smart home data. This work elaborates the comparison done on performance using various algoritms [13]. Shailendra Sing and Shervin Shirmohammadi (2015) discussed about the key elements of power consumption which are related to user activity in real time. Interdepencies of appliancesremains in smart home which stays as a major drawback to determine single appliances consuption. Information of single appliances are extracted using big data prediction [14].

The Proposed work is done by Yi-Cheng Chen, Hsiu-Chieh Hung and Bing-Yang Chiang (2015). Due to the great advent of sensor technology, residents can collect household appliance usage data easily. Prior studies on usage pattern discovery are mainly focused on mining patterns while ignoring the incremental maintenance of mined results. Novel method used is Dynamic Correlation Miner which is developed to incrementally capture and maintain the usage correlations among appliances in a smart home environment. Furthermore, several optimization techniques are used effectively to reduce the search space [15]. The Proposed activity is carried 
out by Charlie Wilson, Lina Stankovic and Michael Coleman (2015). Activities are a descriptive term for the common ways households spend their time on cooking and washing. To generate activities time smart meter data is used that are meaningful to households. Feedbacks from activities are obtained and reported for easy understanding and feature updation needed for elderly people [16].

\section{Summary}

From the above literature survey and comparison table, it is clear that Impulse based model is best suited data clustering algorithm and Semi Markov Model based on Logistic regression is best suited for data classification. The Activity Prediction is done by recognizing human behavior and frequent pattern mining. Mining includes Machine learning algorithms which may be supervised, semi-supervised and unsupervised algorithms. Activity Prediction based pattern mining is temporally analyzed using Semi Markov Model and Impulse Model.

The proposed model is Impulse Based Markov Model where user behavior is predicted as a service based on single appliance applied to real time scenario. Multiple usage predictions are not identified by smart meter at a single time hence smart power plugs can be used. Health care related issues are identified but there is a lack of sending alerts to the patients or care providers which is overcome in the proposed model.

Table 1 shows the parameter comparison of clustering algorithms from literature survey.

\begin{tabular}{|l|c|c|c|c|c|c|}
\hline S. No & Clustering Algorithms & Efficiency & Quality & Robust & Privacy & Security \\
\hline & K-Means [1] & High & Medium & Low & Very High & Medium \\
\hline & Expectation Maximization [3] & High & High & Nil & Nil & Medium \\
\hline & Impulse Based Model [5] & High & High & Medium & High & High \\
\hline & Heuristic Clustering [10,12] & Low & Low & Medium & Medium & Nil \\
\hline & Genetic Algorithm [12,13] & Medium & Medium & Low & Low & Nil \\
\hline
\end{tabular}

Table 1: Parameter Comparison of Clustering Algorithms.

Impulse based markov model

The proposed architecture for health care applications using smart environment big data and human activity patterns is shown in figure 1, obtains smart meter data from database. Then preprocessing data is done for obtaining cleaned integrated data transformed for reduction. Data classification is performed once data preprocessing is done with, classification algorithm used is Semi Markov model based logistic regression.Classified data is clustered using Impulse Model based clustering.

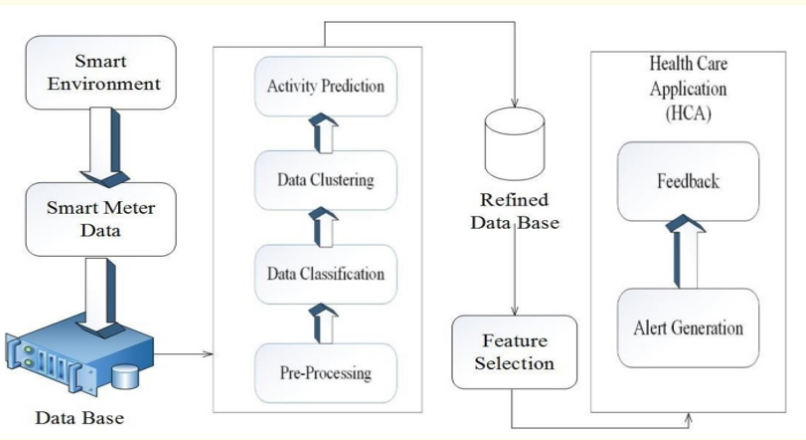

Figure 1: Impulse Based Markov Model.
The clustered data helps in activity prediction and pattern analysis. Data analyzed is made to be stored in database. Alert is immediately generated when activity is predicted in smart environment. By activity prediction and frequent pattern analysis, health care services are provided for the system. Smart Environment is sensed using ambient and mobile sensing and Semi Markov model along with LogisticRegression is used for detecting individual habitant data. Absolute prediction are determined for accuracy and performance. Figure 1 is architecture performed for the proposed model named Impulse based Markov model.

The smart meter is used to measure the appliance usage along with the duration. Using smart meter data, activity recognition and prediction is done in Bayesian network. Data is extracted by which multiple appliance running is identified and temporal energy is analyzed at appliance level. Clustering of data is used for detecting sudden changes in human activity.

Impulse Model based clustering also helps in detecting usage pattern by appliances ON/OFF status. Clustering analysis are used to discover appliance usage based on time. It also includes timestamps.Activity prediction and recognition is done in Bayesian 
network, which uses Directed Acyclic Graph (DAG) and includes the concept of causality. Bayesian network determines mitigating missing data, used to learn relationship between random variables and historical data and variations while overfitting. Accuracy prediction and performance analysis are plotted as graph in figure 2 and 3. The user based items or applicances will have a high performance and accuracy in mining and prediction of data.

Data mining is done for random and popular items that are commonly used appliance in smart home. User based items are like television, washing machine and fan most commonly used items by elders. The feedback is obtained by frequent pattern mining. Alert can be generated each 30 minutes by appliance data fetched from database. Feedback should be provided on the basis of active monitoring. These data helps in improving health care applications. Finally data is integrated to build ontology model displaying their properties and relationship among applicanes.

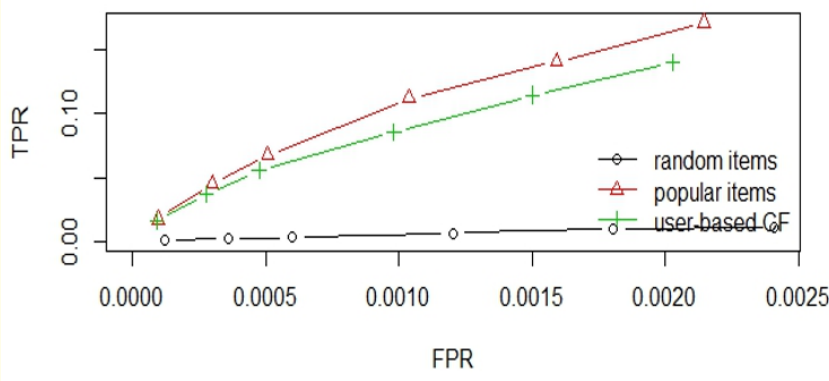

Figure 2: Performance analysis.

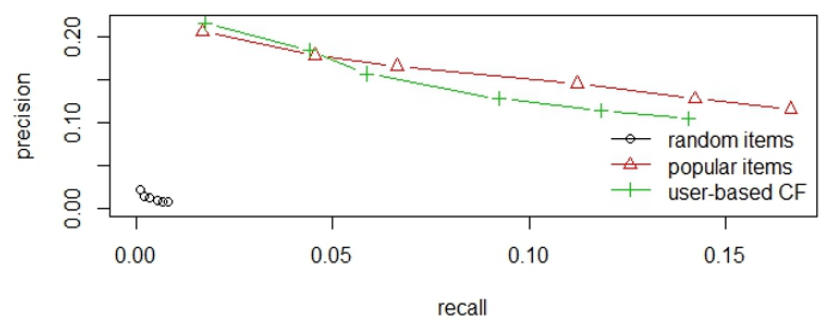

Figure 3: Accuracy prediction.

\section{Conclusion}

Absolute model is presented for recognizing human activity patterns using smart meters whereas pattern mining and prediction are done by user based collaborative filtering method. Ontology model is built to map appliance to activity operating on any quantum of time. Performance and accuracy values are obtained for random, populoar and user based items. Items may vary with appliances. Further enhancement can be made on accuracy on not mostly items of elderky people. Elederly people human activity are monitored in smart home and in case of emergency prediction values are analyzed with mining and results are generated to elders.

\section{Bibliography}

1. Muhammad Aftab and Chi-KinChau. "Smart Power Plugs for Efficient Online Classification and Tracking of Application Behavior". In Proceedings of APSys (2017).

2. Jianguo Hao., et al. "Complex behavioral pattern mining in non-intrusive sensor-based smart Environments using an intelligent activity inference engine". Springer International (2017).

3. Prajakta Joglekar and Vrushali Kulkarni. "Data oriented view of a Smart City a Big Data Approach". International Conference on Emerging Trends and Innovation in ICT (ICEI) (2017).

4. Anish Jindal., et al. "An Efficient Fuzzy Rule-Based Big Data Analytics Scheme for Providing Healthcare-as-a- Service". IEEE ICC SAC Symposium E-Health Track (2017).

5. Abdulsalam Yassine and Atif Alamri. "Mining Human Activity Patterns from Smart Home Big Data for Health Care Applications". IEEE Access (2017).

6. Jaganathan Venkatesh., et al. "Modular and Personalized Smart Health Application Design in a Smart City Environment". IEEE Internet of Things Journal (2017).

7. Md Ileas Pramanik and Md Abul Kalam Azad. "Smart Health: Big Data Enabled Health Paradigm within Smart Cities". Expert Systems with Applications (2017).

8. Min Chen and Lin Wang. "Disease Prediction by Machine Learning Over Big Data from Healthcare Communities". IEEE Access (2017). 
9. M Shamim Hossain., et al. "Cyber Physical Cloud-Oriented Multi-Sensory Smart Home Framework for Elderly People: An Energy Efficiency Perspective". Parallel Distributing Computing Journal (2017).

10. Wen Ming Liu and Lingyu Wang. "Privacy Preserving Smart Meter Streaming against Information Leakage of Appliance Status". IEEE Transaction on Information Forensics and Security (2017).

11. Anthony Faustine., et al. "A Survey on Non-Intrusive Load Monitoring Methodies and Techniques for Energy Disaggregation Problem" (2017).

12. M Victoria Moreno., et al. "Applicability of Big Data Techniques to Smart Cities Deployments". IEEE Transactions on Industrial Informatics (2016).

13. Daniel Schweizer., et al. "Using consumer behavior data to reduce energy consumption in smart homes". International Conference on Machine Learning and Applications (2016).

14. Shailendra Singh., et al. "Incremental Mining of Frequent Power Consumption Patterns from Smart Meters Big Data". IEEE Electrical Power and Energy Conference (EPEC) (2015).

15. Yi-Cheng Chen., et al. "Incrementally Mining Usage Correlations among Appliances in Smart Homes". International Conference on Network Information Systems (2015).

16. Charlie Wilson., et al. "Identifying the time profile of everyday activities in the home using smart meter data". Conference Proceedings (2015).

Volume 3 Issue 8 August 2019

(c) All rights are reserved by $M$ Janani and M Meenalochini. 